\title{
Research on Vibration Suppression Method Based on Coaxial Stacking Measurement
}

\author{
Chuanzhi Sun ${ }^{1,2}$, Ruirui $\mathrm{Li}^{1,2}{ }^{10}$, Ze Chen ${ }^{1,2}$, Yingjie Mei ${ }^{1,2}{ }^{-}$, Xiaoming Wang ${ }^{1,2}$, Chengtian $\mathrm{Li}^{3}$ \\ and Yongmeng Liu 1,2,* \\ 1 Center of Ultra-Precision Optoelectronic Instrument Engineering, Harbin Institute of Technology, \\ Harbin 150001, China; czsun@hit.edu.cn (C.S.); 19B901021@stu.hit.edu.cn (R.L.); \\ 20s001065@stu.hit.edu.cn (Z.C.); 20B901021@stu.hit.edu.cn (Y.M.); wang_xm@hit.edu.cn (X.W.) \\ 2 Key Lab of Ultra-Precision Intelligent Instrumentation Engineering (Harbin Institute of Technology), \\ Ministry of Industry and Information Technology, Harbin 150001, China \\ 3 Reactor Operation and Application Sub-Institute of Nuclear Power Institute of China, \\ Chengdu 610041, China; 20s001021@stu.hit.edu.cn \\ * Correspondence: lym@hit.edu.cn
}

check for updates

Citation: Sun, C.; Li, R.; Chen, Z.;

Mei, Y.; Wang, X.; Li, C.; Liu, Y.

Research on Vibration Suppression Method Based on Coaxial Stacking Measurement. Mathematics 2021, 9, 1438. https://doi.org/10.3390/ math9121438

Academic Editor: Stevan Dubljevic

Received: 30 April 2021

Accepted: 11 June 2021

Published: 21 June 2021

Publisher's Note: MDPI stays neutral with regard to jurisdictional claims in published maps and institutional affiliations.

\begin{abstract}
A dynamic analysis model of the unbalanced vibration response of a single-rotor system is established to study the corresponding mechanism of the unbalanced excitation force and vibration response caused by the deviation of the rotor mass centroid in this paper, and finally to achieve the combined rotor vibration suppression. First, the installation of multi-stage rotors during vibration was studied, and the rotor mass centroid transfer model in the rotating coordinate system was established to obtain the unbalanced excitation force vectors of the rotors at all levels based on the traditional stacking assembly method and axiality measurement. Second, the rotor unbalance excitation force vectors were substituted at all levels to establish the finite element analysis model of the single-rotor system. Finally, a simulation analysis was carried out for the stacking assembly of the three-stage rotor, and the rotor test piece was used for the vibration experiment. The results show that the optimal assembly phase of the multi-stage rotor obtained by the dynamic analysis model of the unbalanced vibration response of the single-rotor system can effectively suppress the vibration of the combined rotor.
\end{abstract}

Keywords: centroid coordinate transformation; double constraint; assembly phase; vibration suppression

\section{Introduction}

In the development and use of advanced rotors, vibration is always one of the main problems [1,2]. According to statistics, more than $60 \%$ of rotor failures are vibration failures. Fifty-six percent of the A-level failures in F100, F110, F404, AL31F, and other third-generation fighters occurred as a result od vibration. The rotor is the core component of an aeroengine, and it is also the key source of vibration for the engine $[3,4]$. Among them, owing to the uneven material of the rotor itself and the asymmetry of the structure, the unbalanced excitation force generated by the rotor centroid shifting the axis of rotation after assembly is an important reason for the vibration of the aero engine [5]. The rotor unbalance excitation force is proportional to the square of the speed. However, continuously increasing the rotor speed is one of the important development trends of rotors [6,7]. Therefore, it is of great significance to the safety, long-term, and high-efficiency work of rotor to achieve combined rotor vibration suppression by adjusting the position of the multi-stage rotor center of mass.

To effectively suppress the vibration caused by the unbalanced excitation force of the rotor, scholars have done a lot of research. Multi-stage rotors are stacked by single-stage rotors. The stacking model is established by studying the error transmission relationship 
of the assembly connection surface, which can control the position of the mass centroid of the rotor at each level to suppress vibration [8]. Zhang put forward a method for modeling geometric form errors of single planes for precision assembly [9]. Li established a multiobjective optimization model of posture adjustment considering engineering constraints including collinear, coplanar, and symmetry constraints and proposed multi-objective optimization algorithm based on the Gauss-Newton method [10]. Sun analyzed the propagation process of geometry and mass eccentric deviations in the multistage rotors assembly based on the connective build model, using spatial error compensation feature to control cumulative eccentricity deviation of multi-stage rotor assembly [11]. Starting from the vibration characteristics, Lusty further considered the flexible behavior of the magnetic actuator support structure based on classic rotor/magnetic bearing systems, designed an experimental facility conforming to the proposed topology, and proposed a vibration control scheme based on $\mathrm{H} \infty$ for this system [12]. Moeenfard applied the intelligent electrorheological elastomer to the field of rotor dynamics, took a Jeffcott rotor model with ER elastomer added at both ends as the research object, used the finite element method based on Rayleigh beam theory to establish the dynamic model, and used a four-parameter viscoelastic model to numerically simulate the ER elastomer support to reduce the vibration level of the rotor system [13].

Based on the above reference, it can be seen that, in the research on indirect realization of multi-stage rotor vibration suppression by establishing the multi-stage rotor error transmission relationship and axiality measurement, optimizing the position of the rotor center of mass at all levels or designing system control methods has been more in-depth. However, in the assembly process of multi-stage rotors, the machining errors of the singlestage rotors are gradually transmitted and amplified through the assembly connection surface. The centroid deviation of the rotors at each stage and the vibration characteristics of the multi-stage rotors after assembly are not clear. Aiming at the actual rotation mode of the multi-stage rotor of rotary parts, through the conversion of the traditional stacking model and the vibration model coordinate system, the center of mass transfer model under the multi-stage rotor rotating coordinate system is established in this paper. Based on the finite element method and the center of mass transfer model in the rotating coordinate system, the dynamic analysis model of the unbalanced vibration response of the singlerotor system is established, and the corresponding mechanism of the unbalanced excitation force and the vibration response generated by the centroid deviation of the rotors at all levels is clear. The rotor vibration suppression is achieved by changing the phase of the rotating rotor.

\section{The Centroid Transfer Model in the Rotation Coordinate System}

As shown in Figure 1, the traditional reference axis $S$ is perpendicular to the bottom face of rotor and cross though the bottom face center $O$. The position vector of mass eccentric deviation for the $k$-th rotor in the assembly using traditional stack-build assembly method [11] can be expressed as

$$
\boldsymbol{d} p_{0-k}^{c}=\left[\begin{array}{c}
d x_{0-k}^{c} \\
d y_{0-k}^{c} \\
d z_{0-k}^{c}
\end{array}\right]=\sum_{i=1}^{k-1}\left(\prod_{j=1}^{i-1}\left(d \boldsymbol{R}_{j}^{r} d \boldsymbol{R}_{j}^{o}\right) d \boldsymbol{R}_{i}^{r} d p_{i}^{o}\right)+\prod_{i=1}^{k-1}\left(d \boldsymbol{R}_{i}^{r} d \boldsymbol{R}_{i}^{o}\right) d \boldsymbol{R}_{k}^{r} d \boldsymbol{p}_{k}^{c}
$$

where $d x_{0-k^{\prime}}^{c} d y_{0-k^{\prime}}^{c}$ and $d z_{0-k}^{c}$ are the cumulative mass eccentric deviations of the $k$-th rotor in the $X, Y$, and $Z$ directions, respectively. $d \boldsymbol{R}_{i}^{r}$ is the rotation matrix of the $i$-th rotor. $\boldsymbol{d} \boldsymbol{R}_{i}^{o}$ is the geometry eccentric deviation matrices of the $i$-th rotor. $d p_{i}^{o}$ and $d p_{i}^{c}$ are the positions of the geometry center and mass center of the $i$-th rotor, respectively. 


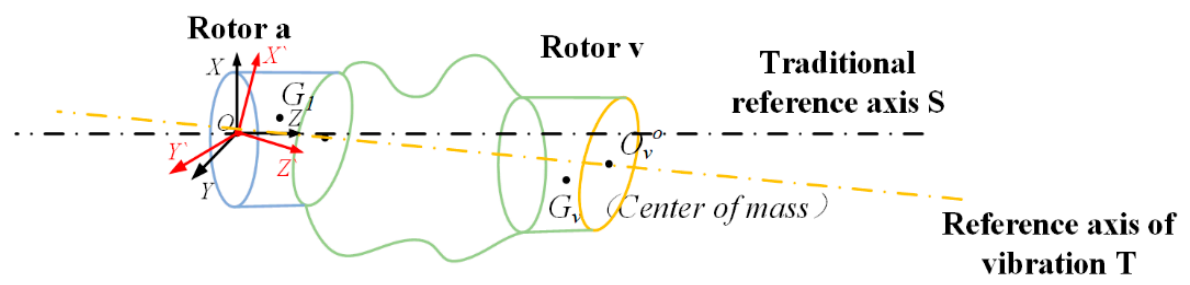

Figure 1. Centroid transfer model in the rotation coordinate system.

In the vibration analysis of the single-rotor system, the connection line between the center $O$ of the lower end surface of the lowest-level rotor a and the center $O_{v}^{o}$ of the upper end surface of the highest-level rotor $v$ is taken as the reference axis $T$. Therefore, when studying the vibration problem caused by the unbalanced excitation force due to the deviation of the rotor center of mass, it is necessary to obtain the coordinates of the rotor mass centroid at various levels in the coordinate system $O X^{\prime} Y^{\prime} Z^{\prime}$. The coordinate system $O X^{\prime} Y^{\prime} Z^{\prime}$ can be regarded as obtained by rotating the coordinate system $O X Y Z$ around the axis passing the origin $\mathrm{O}$ by $\partial$, and setting the rotation axis direction vector 1 to $(1 \mathrm{x}, \mathrm{ly}, \mathrm{lz}) \mathrm{T}$, the unit vector of this vector is $w\left(w_{\mathrm{x}}, w_{\mathrm{y}}, w_{\mathrm{z}}\right) \mathrm{T}=\frac{l}{|l|}$. The vector $l$ and the rotation angle $\partial$ can be obtained by the change of the vector $O O_{v}^{o}$ in the coordinate system $O X Y Z$ and $O X^{\prime} Y^{\prime} Z^{\prime}$, respectively. In the coordinate system $O X Y Z$, the position vector of the measurement surface center of the $k$-th rotor after assembly can be expressed as follows:

$$
\boldsymbol{d} \boldsymbol{p}_{0-k}^{c}=\left[\begin{array}{c}
d x_{0-k}^{o} \\
d y_{0-k}^{o} \\
d z_{0-k}^{o}
\end{array}\right]=\sum_{i=1}^{k}\left(\prod_{j=2}^{i}\left(\boldsymbol{R}_{j-1}^{r} \boldsymbol{R}_{x j-1}^{o} \boldsymbol{R}_{y j-1}^{o}\right) \boldsymbol{R}_{i}^{r}\left(\boldsymbol{p}_{i}^{o}+\boldsymbol{d} \boldsymbol{p}_{i}^{o}\right)\right)
$$

According to Equation (2), the position vector of the center of the circle $O_{v}^{o}$ in the coordinate system $O X Y Z$ is obtained, and the vector $\boldsymbol{P}\left(O_{v x}^{o}, O_{v y}^{o}, O_{v z}^{o}\right)^{T}$ is represented. According to geometric relations, the position vector of center $O_{v}^{o}$ in the coordinate system $O X^{\prime} Y^{\prime} Z^{\prime}$ can be expressed as $Q\left(0,0, O_{v z}^{\prime o}\right)^{T}$, where $O_{v z}^{\prime o}=\sqrt{\left(O_{v z}^{o}\right)^{2}+\left(O_{v x}^{o}\right)^{2}+\left(O_{v y}^{o}\right)^{2}}$, then the direction vector 1 of the rotation axis and the rotation angle $\partial$ are shown in Equations (3) and (4):

$$
\begin{gathered}
\boldsymbol{l}=\boldsymbol{P} \times \boldsymbol{Q}=\left[\begin{array}{c}
O_{v y}^{o}{O^{\prime o}}_{v z}^{o} \\
-O_{v x}^{o}{O^{\prime o}}_{v z} \\
0
\end{array}\right] \\
\theta=\arccos \left(\frac{\boldsymbol{P} \cdot \boldsymbol{Q}}{|\boldsymbol{P}||\boldsymbol{Q}|}\right)
\end{gathered}
$$

The rotation matrix $\mathrm{A}$ is represented by the unit vector $\mathrm{w}$ of rotation axis and rotation angle $\partial$, as shown in Equation (5):

$$
\boldsymbol{A}=\left[\begin{array}{ccc}
\cos \partial+w_{x}^{2}(1-\cos \partial) & w_{x} w_{y}(1-\cos \partial)-w_{z} \sin \partial & w_{y} \sin \partial+w_{x} w_{z}(1-\cos \partial) \\
w_{z} \sin \partial+w_{x} w_{y}(1-\cos \partial) & \cos \partial+w_{y}^{2}(1-\cos \partial) & -w_{x} \sin \partial+w_{y} w_{z}(1-\cos \partial) \\
-w_{y} \sin \partial+w_{x} w_{z}(1-\cos \partial) & w_{x} \sin \partial+w_{y} w_{z}(1-\cos \partial) & \cos \partial+w_{z}^{2}(1-\cos \partial)
\end{array}\right]
$$

Suppose the position vector of the mass center of the $k$-th rotor in the coordinate system $O X^{\prime} Y^{\prime} Z^{\prime}$ is $\left(d x_{0-k}^{\prime c}, d y_{0-k}^{\prime c}, d z_{0-k}^{\prime c}\right)^{\mathrm{T}}$, according to the coordinate conversion relationship, then,

$$
\left[\begin{array}{l}
d x_{0-k}^{\prime c} \\
d y_{0-k}^{\prime c} \\
d z_{0-k}^{\prime c}
\end{array}\right]=A\left[\begin{array}{c}
d x_{0-k}^{c} \\
d y_{0-k}^{c} \\
d z_{0-k}^{c}
\end{array}\right]
$$


When the rotational angular velocity of the rotor is $\Omega$, and the mass of the $k$-th rotor is set to $m_{k}$, the unbalanced excitation force $Q^{r}$ of the $k$-th rotor in the coordinate system $O X^{\prime} Y^{\prime} Z^{\prime}$ due to the offset of the center of mass is shown in Equation (7) [14]:

$$
Q^{r}=m_{k} \Omega^{2}\left[\begin{array}{c}
d x_{0-k}^{\prime c} \\
d y_{0-k}^{\prime c} \\
0 \\
0
\end{array}\right] \cos \Omega t+m_{k} \Omega^{2}\left[\begin{array}{c}
-d y^{\prime c}{ }^{2} \\
d x_{0-k}^{\prime c} \\
0 \\
0
\end{array}\right] \sin \Omega t
$$

\section{Finite Element Analysis Model of Single Rotor System}

To study the influence mechanism of the unbalanced rotor excitation force and the unbalanced vibration response of the system, the finite element method was used for analysis. This method simplifies the rotor system into discrete rigid disks (rotors at all levels), elastic shaft segments, and bearings with stiffness and damping. The rotor system motion equation is synthesized by the motion equations of the above elements $[14,15]$.

\subsection{Equation of Motion of Shaft Element}

When applying the finite element method [16-18] for analysis, the shaft element is usually set as Timoshenko beam. As Figure 2 shows, each shaft element has two front and rear nodes, and each node has four degrees of freedom (two translations $V$ and $W$, and two rotations $B$ and $F$ ), so each element has a total of eight degrees of freedom. The generalized

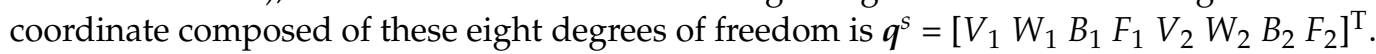

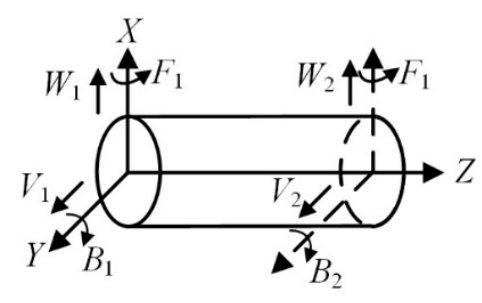

Figure 2. Finite element analysis.

The motion equation of the shaft element is shown in Equation (8):

$$
\left(\boldsymbol{M}_{T}^{s}+\boldsymbol{M}_{R}^{s}\right) \ddot{\boldsymbol{q}}^{s}-\Omega \boldsymbol{G}^{s} \dot{\boldsymbol{q}}^{s}+\boldsymbol{K}^{s} \boldsymbol{q}^{s}=\boldsymbol{Q}^{s}
$$

where $\boldsymbol{M}_{T}^{s}$ and $\boldsymbol{M}_{R}^{s}$ are the translatory and rotary inertia matrices of the shaft mass. $\boldsymbol{G}^{s}$ is the gyroscopic matrix of the shaft. $K_{B}^{s}$ is the stiffness matrix. $Q^{s}$ is the generalized force vector of the shaft element.

$$
\boldsymbol{M}_{T}^{s}=\frac{\rho_{l} l}{\left(1+\varphi_{s}\right)^{2}}\left[\begin{array}{cccccccc}
M_{T 1} & 0 & 0 & M_{T 4} & M_{T 3} & 0 & 0 & -M_{T 5} \\
0 & M_{T 1} & -M_{T 4} & 0 & 0 & M_{T 3} & M_{T 5} & 0 \\
0 & -M_{T 4} & M_{T 2} & 0 & 0 & -M_{T 5} & M_{T 6} & 0 \\
M_{T 4} & 0 & 0 & M_{T 2} & M_{T 5} & 0 & 0 & -M_{T 4} \\
M_{T 3} & 0 & 0 & M_{T 5} & M_{T 1} & 0 & 0 & -M_{T 4} \\
0 & M_{T 3} & -M_{T 5} & 0 & 0 & M_{T 1} & M_{T 4} & 0 \\
0 & M_{T 5} & M_{T 6} & 0 & 0 & M_{T 4} & M_{T 2} & 0 \\
-M_{T 5} & 0 & 0 & M_{T 6} & -M_{T 4} & 0 & 0 & M_{T 2}
\end{array}\right]
$$


where $M_{T 1}=\frac{13}{35}+\frac{7}{10} \varphi_{s}+\frac{1}{3} \varphi_{s}^{2}, M_{T 2}=\left(\frac{1}{105}+\frac{1}{60} \varphi_{s}+\frac{1}{120} \varphi_{s}^{2}\right) l^{2}, M_{T 3}=\frac{9}{10}+$ $\frac{3}{10} \varphi_{s}+\frac{1}{6} \varphi_{s}^{2}, \quad M_{T 4}=\left(\frac{11}{210}+\frac{11}{120} \varphi_{s}+\frac{1}{24} \varphi_{s}^{2}\right) l, \quad M_{T 5}=\left(\frac{13}{420}+\frac{3}{40} \varphi_{s}+\frac{1}{24} \varphi_{s}^{2}\right) l$, $M_{T 6}=\left(\frac{1}{140}+\frac{1}{60} \varphi_{s}+\frac{1}{120} \varphi_{s}^{2}\right) l^{2}$.

$\boldsymbol{M}_{R}^{s}=\frac{\rho_{l} I}{l\left(1+\varphi_{s}\right)^{2} A}\left[\begin{array}{cccccccc}M_{R 1} & 0 & 0 & M_{R 4} & -M_{R 1} & 0 & 0 & M_{R 4} \\ 0 & M_{R 1} & -M_{R 4} & 0 & 0 & -M_{R 1} & -M_{R 4} & 0 \\ 0 & -M_{R 4} & M_{R 2} & 0 & 0 & M_{R 4} & M_{R 3} & 0 \\ M_{R 4} & 0 & 0 & M_{R 2} & -M_{R 4} & 0 & 0 & M_{R 3} \\ -M_{R 1} & 0 & 0 & -M_{R 4} & M_{R 1} & 0 & 0 & -M_{R 4} \\ 0 & -M_{R 1} & M_{R 4} & 0 & 0 & M_{R 1} & M_{R 4} & 0 \\ 0 & -M_{R 4} & M_{R 3} & 0 & 0 & M_{R 4} & M_{R 2} & 0 \\ M_{R 4} & 0 & 0 & M_{R 3} & -M_{R 4} & 0 & 0 & M_{R 2}\end{array}\right]$ where $M_{R 1}=\frac{6}{5}, M_{R 2}=\left(\frac{2}{15}+\frac{1}{6} \varphi_{s}+\frac{1}{3} \varphi_{s}^{2}\right) l^{2}, M_{R 3}=\left(-\frac{1}{30}-\frac{1}{6} \varphi_{s}+\frac{1}{6} \varphi_{s}^{2}\right) l^{2}$, $M_{R 4}=\left(\frac{1}{10}-\frac{1}{2} \varphi_{s}\right) l$.

$$
G^{s}=\frac{\rho_{l} I}{15 l\left(1+\varphi_{s}\right)^{2} A}\left[\begin{array}{cccccccc}
0 & -G_{1} & G_{2} & 0 & 0 & G_{1} & G_{2} & 0 \\
G_{1} & 0 & 0 & G_{2} & -G_{1} & 0 & 0 & G_{2} \\
-G_{2} & 0 & 0 & -G_{4} & G_{2} & 0 & 0 & G_{3} \\
0 & -G_{2} & G_{4} & 0 & 0 & G_{2} & -G_{3} & 0 \\
0 & G_{1} & -G_{2} & 0 & 0 & -G_{1} & -G_{2} & 0 \\
-G_{1} & 0 & 0 & -G_{2} & G_{1} & 0 & 0 & -G_{2} \\
-G_{2} & 0 & 0 & G_{3} & G_{2} & 0 & 0 & -G_{4} \\
0 & -G_{2} & -G_{3} & 0 & 0 & G_{2} & G_{4} & 0
\end{array}\right]
$$

where $G_{1}=36, G_{2}=3 l-15 l \varphi_{s}, G_{3}=l^{2}+5 l^{2} \varphi_{s}-5 l^{2} \varphi_{s}^{2}, G_{4}=4 l^{2}+5 l^{2} \varphi_{s}+10 l^{2} \varphi_{s}^{2}$

$$
\boldsymbol{K}^{S}=\frac{E I}{l^{3}\left(1+\varphi_{s}\right)}\left[\begin{array}{cccccccc}
K_{1} & 0 & 0 & K_{4} & -K_{1} & 0 & 0 & K_{4} \\
0 & K_{1} & -K_{4} & 0 & 0 & -K_{1} & -K_{4} & 0 \\
0 & -K_{4} & K_{2} & 0 & 0 & K_{4} & K_{3} & 0 \\
K_{4} & 0 & 0 & K_{2} & -K_{4} & 0 & 0 & K_{3} \\
-K_{1} & 0 & 0 & -K_{4} & K_{1} & 0 & 0 & -K_{4} \\
0 & -K_{1} & K_{4} & 0 & 0 & K_{1} & -K_{4} & 0 \\
0 & -K_{4} & K_{3} & 0 & 0 & -K_{4} & K_{2} & 0 \\
K_{4} & 0 & 0 & K_{3} & -K_{4} & 0 & 0 & K_{2}
\end{array}\right]
$$

where $K_{1}=12, K_{2}=\left(4+\varphi_{s}\right) l^{2}, K_{3}=\left(2-\varphi_{s}\right) l^{2}, K_{4}=6 l$.

And $\rho_{l}, l, \varphi_{s}, I, A$, and $E$ denote the density, length, shear deformation coefficient, section moment of inertia, section area, and elastic modulus of the shaft element respectively.

\subsection{Equation of Motion of Disk (Rotor) Element}

Each disk is considered as a rigid element with concentrated inertial properties and mass unbalance. The motion equation of the disc element is shown in Equation (9):

$$
\left(\boldsymbol{M}_{T}^{d}+\boldsymbol{M}_{R}^{d}\right) \ddot{\boldsymbol{q}}^{d}-\Omega \boldsymbol{G}^{d} \dot{\boldsymbol{q}}^{d}=\boldsymbol{Q}^{d}
$$

where $\boldsymbol{M}_{T}^{d}$ and $\boldsymbol{M}_{R}^{d}$ are the translatory and rotary inertia matrices of the disk mass. $G^{d}$ is the gyroscopic matrix of the disk element. $q^{d}$ is the generalized displacement vector of the rotor, $\boldsymbol{q}^{d}=\left[\begin{array}{llll}V & W & B & F\end{array}\right]^{\mathrm{T}}$. $Q^{d}$ is the external force vector on the disk. 
Each stage of the rotor in the previous section can be regarded as a disk element located at a fixed node of the finite element grid, and the external force vector $Q^{d}$ can be calculated by Equation (7).

$$
\boldsymbol{M}_{T}^{d}=\left[\begin{array}{cccc}
m_{d} & 0 & 0 & 0 \\
0 & m_{d} & 0 & 0 \\
0 & 0 & 0 & 0 \\
0 & 0 & 0 & 0
\end{array}\right] \boldsymbol{M}_{R}^{d}=\left[\begin{array}{cccc}
0 & 0 & 0 & 0 \\
0 & 0 & 0 & 0 \\
0 & 0 & I_{d} & 0 \\
0 & 0 & 0 & I_{d}
\end{array}\right] \boldsymbol{G}^{d}=\left[\begin{array}{cccc}
0 & 0 & 0 & 0 \\
0 & 0 & 0 & 0 \\
0 & 0 & 0 & -I_{p} \\
0 & 0 & I_{p} & 0
\end{array}\right]
$$

where $m_{d}, I_{d}$, and $I_{p}$ are the mass, diametral moment of inertia, and polar mass moment of inertia for the corresponding rigid disk, respectively.

\subsection{Equation of Motion of Bearing Element}

When considering linear stiffness and damping, the motion equation of the bearing is as follows:

$$
\boldsymbol{C}^{b} \dot{\boldsymbol{q}}^{b}+\boldsymbol{K}^{b} \boldsymbol{q}^{b}=Q^{b}
$$

where $C^{b}$ is bearing damping matrix, $K^{b}$ is bearing stiffness matrix, $Q^{b}$ is the external force vector of the bearing, $q^{b}$ is the generalized displacement vector of the bearing, and $\boldsymbol{q}^{b}=\left[\begin{array}{llll}V & W & B & F\end{array}\right]^{\mathrm{T}}$.

$$
\boldsymbol{C}^{b}=\left[\begin{array}{cccc}
c_{y y}^{b} & c_{y z}^{b} & 0 & 0 \\
c_{z y}^{b} & c_{z z}^{b} & 0 & 0 \\
0 & 0 & 0 & 0 \\
0 & 0 & 0 & 0
\end{array}\right] \boldsymbol{K}^{b}=\left[\begin{array}{cccc}
k_{y y}^{b} & k_{y z}^{b} & 0 & 0 \\
k_{z y}^{b} & k_{z z}^{b} & 0 & 0 \\
0 & 0 & 0 & 0 \\
0 & 0 & 0 & 0
\end{array}\right]
$$

Assuming that the bearing is isotropic, then $k_{y z}=k_{z y}=c_{y z}=c_{y z}=0$ and $k_{y y}=k_{z z}$, $c_{y y}=c_{z z}$.

\subsection{Equation of Motion of the Rotor System}

According to the characteristics of the rotor system, the system is divided into $n-1$ units and $n$ nodes, then the generalized displacement vector $\boldsymbol{q}$ of the entire rotor system can be expressed as follows:

$\boldsymbol{q}=\left[\begin{array}{lllllllllllllll}V_{1} & W_{1} & B_{1} & F_{1} & \cdots & V_{i} & W_{i} & B_{i} & F_{i} & \cdots & V_{n} & W_{n} & B_{n} & F_{n} & \end{array}\right]^{\mathrm{T}}$

Add the coefficient matrices of the similar items in the motion equations of the disk element, the shaft element, and the bearing element to obtain the steady-state motion equation of the rotor system, as shown in the following equation:

$$
M \ddot{q}+(C-\Omega G) \dot{q}+K \boldsymbol{q}=Q
$$

where $M$ is the mass assembly matrix, which contains the translational and rotary effects of the shaft element and the disk element. Just like $M, G$ is the gyroscopic assembly matrix that considers the effects of the shaft element and the disk element. $C$ is the damping assembly matrix. $K$ is the stiffness assembly matrix that considers the stiffness effects of the shaft element and the disk element. $Q$ is the excitation matrix. To study the influence of the unbalanced excitation force caused by the deviation of the center of mass on the rotor system, $Q$ only considers the unbalanced excitation force after the multi-stage rotor is stacked and assembled.

\subsection{Unbalanced Response Solution}

The unbalanced force of the system can be expressed as follows:

$$
Q=Q_{c} \cos \Omega t+Q_{s} \sin \Omega t
$$


Then, the steady-state solution of the differential equation of motion of the rotor system can be set as follows:

$$
\boldsymbol{q}=\boldsymbol{q}_{c} \cos \Omega t+\boldsymbol{q}_{s} \sin \Omega t
$$

Substituting Equation (14) into Equation (12), derivation, and sorting can obtain the following:

$$
\left[\begin{array}{l}
\boldsymbol{q}_{c} \\
\boldsymbol{q}_{s}
\end{array}\right]=\left[\begin{array}{cc}
\boldsymbol{K}-\boldsymbol{M} \Omega^{2} & \Omega(\boldsymbol{C}-\Omega \boldsymbol{G}) \\
-\Omega(\boldsymbol{C}-\Omega \boldsymbol{G}) & \boldsymbol{K}-\boldsymbol{M} \Omega^{2}
\end{array}\right]^{-1}\left[\begin{array}{l}
\boldsymbol{Q}_{\mathrm{c}} \\
\boldsymbol{Q}_{s}
\end{array}\right]
$$

Then, the unbalanced response is as follows:

$$
\boldsymbol{q}=\boldsymbol{q}^{\prime} \cos (\Omega t-\theta)
$$

where

$$
\left\{\begin{array}{c}
\boldsymbol{q}^{\prime}=\sqrt{\boldsymbol{q}_{c}^{2}+\boldsymbol{q}_{s}^{2}} \\
\boldsymbol{\theta}=\arctan \frac{\boldsymbol{q}_{s}}{\boldsymbol{q}_{c}}
\end{array}\right.
$$

\section{Simulation Analysis}

To study that the unbalanced vibration response dynamic analysis model established in this paper can realize the effectiveness of vibration suppression after assembly of the multi-stage rotor, a simulation analysis is carried out for the stacked assembly of the three-stage rotor. Assuming that the masses of the three-stage rotor are $27.4 \mathrm{~kg}, 20 \mathrm{~kg}$, and $58 \mathrm{~kg}$, respectively, the height of the three-stage rotor is $120 \mathrm{~mm}, 130 \mathrm{~mm}$, and $240 \mathrm{~mm}$, respectively; the mass eccentricity error of each rotor in the $X, Y$, and $Z$ axis directions is $0.02 \mathrm{~mm}$; the geometric eccentricity error of the radial measuring surface of each rotor in the $X$ and $Y$ axis directions is $0.04 \mathrm{~mm}$; the verticality of the axial measuring surface of the rotors at all levels is $0.005 \mathrm{~mm}$; and the angle between the center of the measuring surface of each rotor and the $X$ axis is $0^{\circ}$ when it points to the lowest sampling point. After the rotor is assembled, elastic supports are applied at the front and rear journals. The front support rigidity is $0.8 \times 10^{7} \mathrm{~N} / \mathrm{m}$, the rear support rigidity is $1.6 \times 10^{7} \mathrm{~N} / \mathrm{m}$, and the working speed is $10,000 \mathrm{rpm}$.

Figure 3 is a diagram showing the vibration amplitude of the corresponding node of the rear support after adjusting the second-stage rotor installation phase $\theta_{\mathrm{r} 2}$ and the third-stage rotor installation phase $\theta_{\mathrm{r} 3}$ at the working speed of the rotor system assembled with the three-stage rotor. Analyzing Figure 3 shows that, at the same speed, adjusting the installation phase of the rotors at all levels will change the vibration response. When the second-stage rotor installation phase $\theta_{\mathrm{r} 2}$ is $195^{\circ}$ and the third-stage rotor installation phase $\theta_{\mathrm{r} 3}$ is $0^{\circ}$, the maximum vibration response amplitude is $27.1 \mu \mathrm{m}$; when $\theta_{\mathrm{r} 2}$ is $180^{\circ}$ and $\theta_{\mathrm{r} 3}$ is $180^{\circ}$, the minimum vibration response amplitude is $2.9 \mu \mathrm{m}$; compared with the worst assembly, the vibration amplitude is optimized by $89 \%$ in the optimal assembly. Therefore, the optimal assembly phase of the rotor can be obtained through the dynamic analysis model of the unbalanced vibration response of the multi-stage rotor after assembly established in this paper, and the vibration suppression of the combined rotor can be achieved.

Figure 4 is a graph of the vibration amplitude of the corresponding nodes of the rear support when the rotation speed is increased from 0 to $15,000 \mathrm{rpm}$, and the rotor system of the three-stage rotor assembly is the optimal assembly and the direct assembly, respectively. Amplitude and minimum rotor assembly phase correspond to the optimal assembly when the rotation speed is $1500 \mathrm{rpm}, 5000 \mathrm{rpm}$, and 10,000 rpm. The assembly method is direct assembly when the assembly angle of each rotor is $0^{\circ}$ and no assembly phase adjustment is performed. It can be seen from Figure 4 that, when the rotation speed increases from 0 to $3000 \mathrm{rpm}$, the vibration response amplitude increases continuously, and the vibration amplitude increase rate of the corresponding node of the directly assembled rear support 
is much higher than that of the optimal assembly. When the rotation speed is $3000 \mathrm{rpm}$, the vibration amplitude corresponding to the direct assembly and the optimal assembly has the first peak value, which is $47.3 \mu \mathrm{m}$ and $3.5 \mu \mathrm{m}$, respectively. As the speed continues to increase, the vibration amplitude first decreases and then increases, and the second peak appears at $7000 \mathrm{rpm}$. At this time, the vibration amplitudes corresponding to direct assembly and optimal assembly are $17.6 \mu \mathrm{m}$ and $11.3 \mu \mathrm{m}$, respectively. When the rotation speed reaches 10,000 rpm, the vibration amplitudes corresponding to direct assembly and optimal assembly are $6.6 \mu \mathrm{m}$ and $2.9 \mu \mathrm{m}$, respectively, and the vibration amplitude is optimized by $56 \%$. When the rotation speed is greater than the working rotation speed of 10,000 rpm, the vibration amplitude changes slightly with the increase of the rotation speed and tends to be stable. Therefore, compared with the direct assembly, the optimal assembly can greatly suppress the vibration of the rotor system.

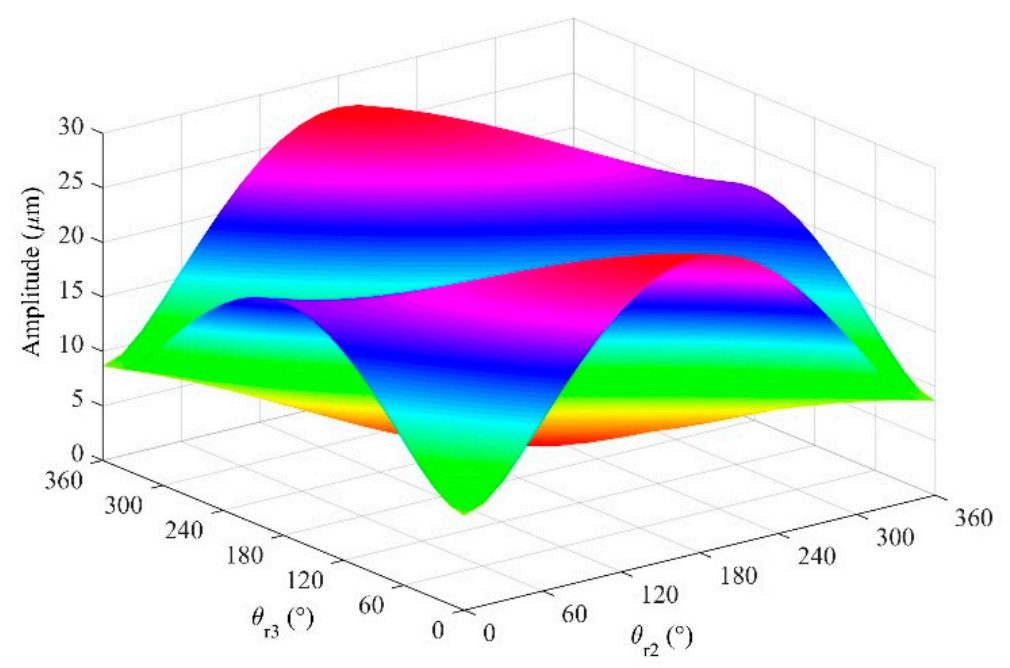

Figure 3. Corresponding vibration amplitude diagrams at the rear support under different assembly phases.

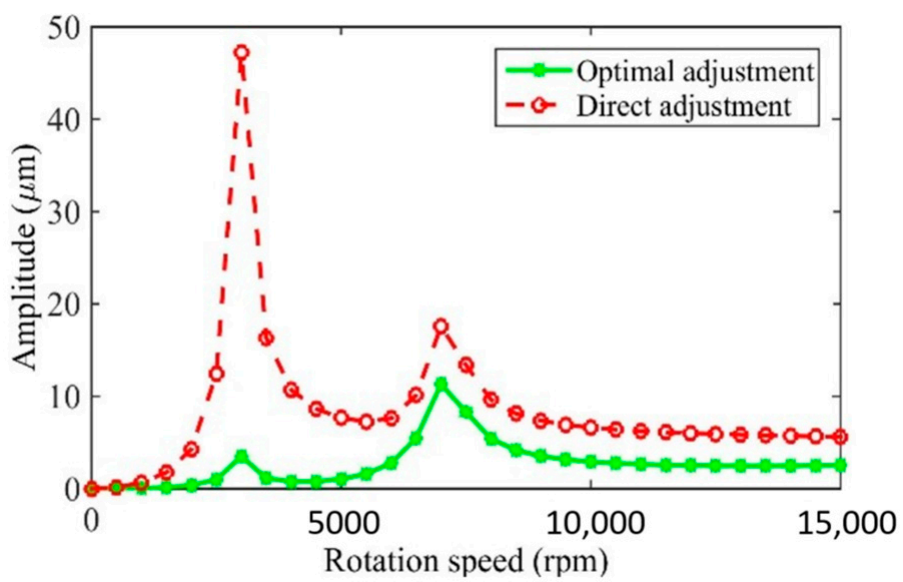

Figure 4. Corresponding vibration amplitude diagram at the rear support at different speeds.

\section{Experimental Verification}

To verify the effectiveness of the unbalanced vibration response dynamics analysis model of the single-rotor system established in this paper in suppressing the vibration of the combined rotor, the rotor test piece shown in Figure 5 a was used for vibration experiments. 


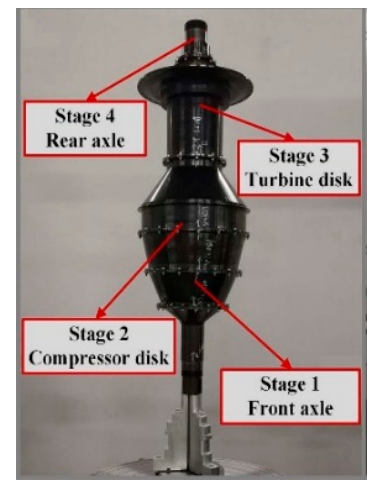

(a)

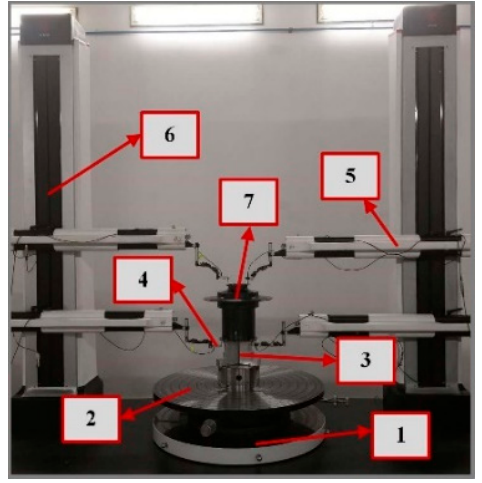

(b)

Figure 5. Rotor geometric parameter measuring device: (a) four rotors assembly; (b) rotary measuring instrument.

First, use the double-column Rotary parts ultra-precision assembly measuring instrument shown in Figure $5 b$ to measure the geometric error parameters of the single-stage rotor. The parameters of each core unit of the measuring instrument are as follows:

(1) Air-bearing turntable is used to provide the rotary measurement datum. The radial and axial accuracies of air-bearing turntable are $80 \mathrm{~nm}$.

(2) Centering and tilt worktable is used for adjusting the eccentricity and tilt of base surface of the rotors to make the geometric axis coincident with the rotation axis. The minimum adjustments of displacement and angle are $0.2 \mu \mathrm{m}$ and $0.2^{\prime \prime}$, respectively.

(3) The chuck is used to fix the rotor.

(4) The inductive sensors are used to collect the radial and axial surface data of the rotors, of which the resolutions are $0.1 \mu \mathrm{m}$.

(5) The displacement of the horizontal guide rail is $800 \mathrm{~mm}$.

(6) The displacement of the vertical guide rail is $2000 \mathrm{~mm}$.

(7) The turbine disk is the measured rotor.

The geometric characteristic parameters of the rotor components at all levels are shown in Table 1.

Table 1. Geometric characteristics and error parameters of the rotor experimental parts.

\begin{tabular}{|c|c|c|c|c|}
\hline Part Name & Front Axle & Compressor Disk & Turbine Disk & Rear Axle \\
\hline Radius of upper end face (mm) & 43 & 189 & 100 & 66 \\
\hline Radius of lower end face (mm) & 189 & 100 & 66 & 40 \\
\hline Height (mm) & 560 & 245 & 325.5 & 124.5 \\
\hline Geometric eccentricity error in $\mathrm{d}_{\mathrm{x}}{ }^{\mathrm{o}}(\mathrm{mm})$ & 0.0026 & 0.0011 & -0.0119 & -0.0012 \\
\hline Geometric eccentricity error in $\mathrm{d}_{\mathrm{y}}{ }^{\mathrm{o}}(\mathrm{mm})$ & 0.0419 & 0.0051 & -0.0047 & 0.0009 \\
\hline Geometric eccentricity error in $\mathrm{d}_{\mathrm{z}}{ }^{\mathrm{o}}(\mathrm{mm})$ & 0.0021 & 0.0015 & 0.0003 & 0.0029 \\
\hline Tilt error $\theta x(")$ & -1.5 & -2 & 0.7 & -7.7 \\
\hline Tilt error $\theta y(")$ & -1.8 & -2.3 & 0.6 & -12.8 \\
\hline Tilt angel $\theta \mathrm{t}\left({ }^{\prime \prime}\right)$ & 2.3 & 3.1 & 0.9 & 15 \\
\hline Lowest point of tilt $\theta 1\left(^{\circ}\right)$ & 220 & 221 & 51 & 221 \\
\hline
\end{tabular}

The parameters are shown in Table 2. 
Table 2. The quality characteristics and error parameters of the rotor experimental parts.

\begin{tabular}{ccccc}
\hline Part Name & Front Axle & Compressor Disk & Turbine Disk & Rear Axle \\
\hline mass $(\mathrm{g})$ & 27,850 & 19,278 & 53,278 & 5032 \\
\hline Mass eccentricity error $\mathrm{d}_{\mathrm{x}}{ }^{\mathrm{c}}(\mathrm{mm})$ & 0.0042 & -0.0080 & -0.0042 & 0.0124 \\
\hline Mass eccentricity error $\mathrm{d}_{\mathrm{y}}{ }^{\mathrm{c}}(\mathrm{mm})$ & -0.0039 & -0.0067 & 0.0041 & 0.0111 \\
\hline Mass eccentricity error $\mathrm{d}_{\mathrm{z}}{ }^{\mathrm{c}}(\mathrm{mm})$ & 380 & 91 & 218 & 51 \\
\hline
\end{tabular}

According to the dynamic analysis model of unbalanced vibration response of singlerotor system established in this paper, the optimally assembled rotor assembly phase angle is obtained. The rotor test pieces were assembled according to the direct assembly and optimal assembly strategies respectively, and the test pieces were installed on the dynamic vibration measurement test bench as shown in Figure 6 for experiments. The dynamic vibration measurement test bench includes a basic platform, a speed-increasing drive system, a test system, a lubrication system, a support system, and a rotor test piece system.

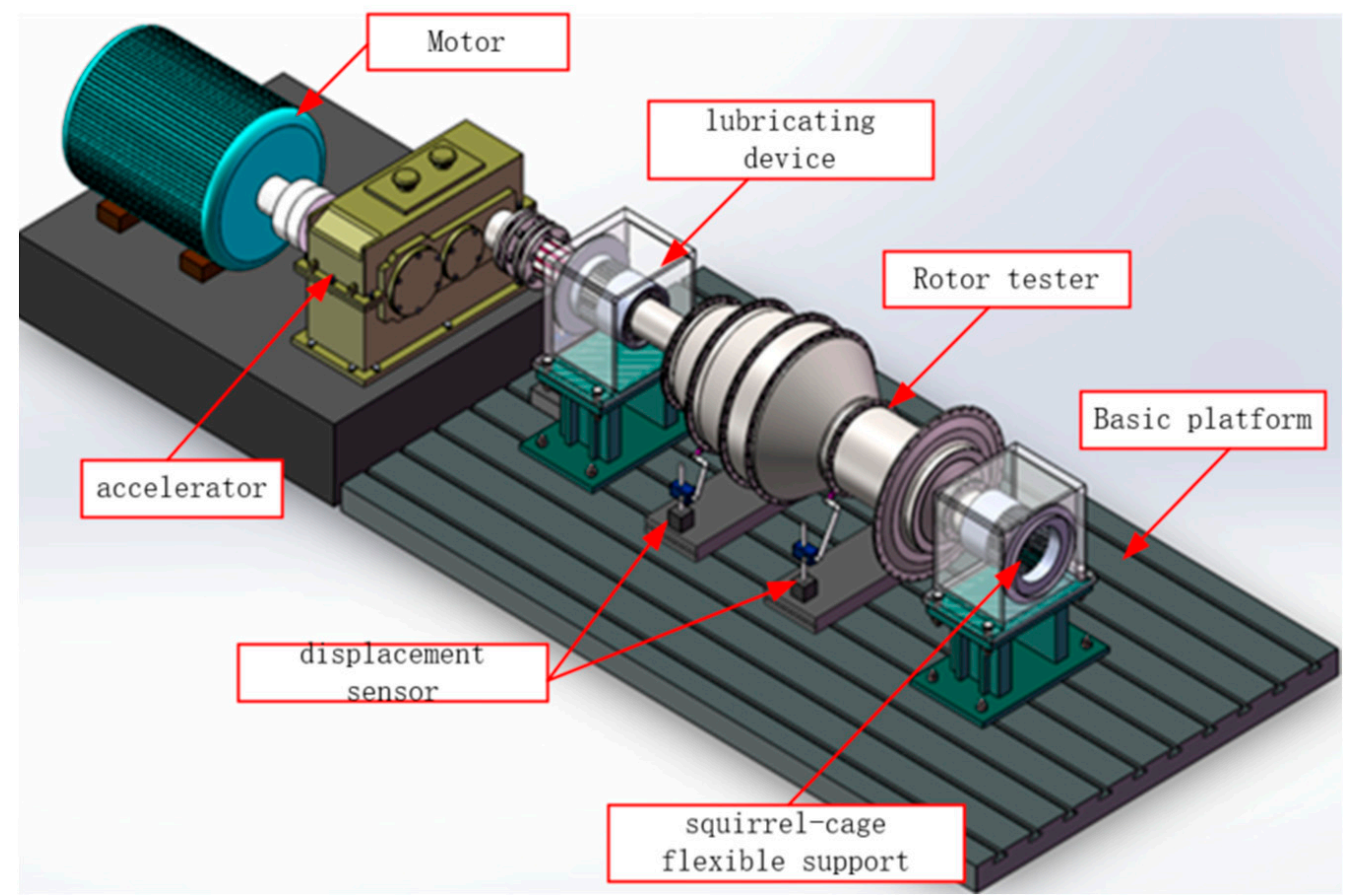

Figure 6. Dynamic vibration measurement test bench.

Compare the actual measured values of the vibration response amplitude of the front support of the combined rotor turbine disc under the direct assembly and the optimal assembly strategy, then record them in Table 3. It can be seen from the data in the table that the vibration amplitude of the rotor experimental part under the optimal assembly strategy is smaller than the vibration amplitude of the directly assembled rotor experimental part. The vibration response of the assembled rotor experimental part is shown when the speed is $3000 \mathrm{rpm}, 6000 \mathrm{rpm}$, and $9000 \mathrm{rpm}$. The amplitude decreased by $29.8 \%, 20.9 \%$, and $22.0 \%$, with an average decrease of $23.8 \%$. Therefore, the dynamic analysis model of unbalanced vibration response of single-rotor system established in this paper can effectively suppress rotor vibration. 
Table 3. Comparison of amplitude measurement results of rotor experimental parts under different assembly strategies.

\begin{tabular}{cccc}
\hline & $\mathbf{3 0 0 0} \mathbf{~ r p m}$ & $\mathbf{6 0 0 0} \mathbf{~ r p m}$ & $\mathbf{9 0 0 0 ~ \mathbf { ~ p m }}$ \\
\hline Optimal assembly & $7.4 \mu \mathrm{m}$ & $13.1 \mu \mathrm{m}$ & $9.2 \mu \mathrm{m}$ \\
\hline Direct assembly & $10.5 \mu \mathrm{m}$ & $16.3 \mu \mathrm{m}$ & $11.8 \mu \mathrm{m}$ \\
\hline Optimization effect & $29.8 \%$ & $19.6 \%$ & $22.0 \%$ \\
\hline
\end{tabular}

\section{Conclusions and Discussion}

To study the corresponding mechanism between the unbalanced excitation force and the vibration response caused by the deviation of the rotor center of mass, the dynamic analysis model of the unbalanced vibration response of the single-rotor system was established in this paper. Through finite element simulation, the vibration amplitude of each node of the rotor at different speeds is obtained. The conclusion is summarized as follows:

(1) From the center of mass transfer model, it can be seen that the rotor vibration amplitude is affected by the installation phase of the rotor at all levels. The dynamic analysis model of the unbalanced vibration response of the single-rotor system established in this paper can directly reflect the corresponding relationship between the rotor installation phase and the vibration amplitude at different speeds.

(2) According to the dynamic analysis model of the unbalanced vibration response of the single-rotor system, the optimal assembly phase of the multi-stage rotor can be obtained, and then the unbalanced excitation force of the multi-stage rotor can be adjusted to achieve the combined rotor vibration suppression.

(3) When the speed is $3000 \mathrm{rpm}$, the uncertainty introduced by measurement repeatability $u\left(x_{1}\right)=1.00 \mu \mathrm{m}$, the uncertainty introduced by the resolution of eddy current sensor $u\left(x_{2}\right)=0.01 \mu \mathrm{m}$, and the standard uncertainty is $\mathrm{U}=2.00 \mu \mathrm{m}(k=2)$.

(4) It is verified by the vibration experiment of the rotor test piece that the optimal assembly phase obtained by the dynamic analysis model of the unbalanced vibration response of the single-rotor system can effectively suppress the vibration of the combined rotor.

The focus of this paper is to achieve rotor vibration suppression by studying the corresponding mechanism between the unbalanced excitation force and vibration response caused by the deviation of the rotor center of mass. In future work, attention can be focused on studying whether there is a cancellation feature between the unbalanced excitation force vectors generated by the rotor centroid deviation at all levels and, if this feature exists, whether the feature can be used to guide the rotor design process.

Author Contributions: Conceptualization, Y.L.; methodology R.L.; experiment, Z.C. and C.L. software and validation, C.S. and Y.M.; investigation, X.W. All authors have read and agreed to the published version of the manuscript.

Funding: This work was supported by the National Natural Science Foundation major research projects of China (grant number 91960109), the National Natural Science Foundation of China (grant number 51805117), the China postal Postdoctoral Science Foundation (grant number 2019M651279), and the Heilongjiang Postdoctoral Fund (grant number LBH-Z18078).

Institutional Review Board Statement: Not applicable.

Informed Consent Statement: Not applicable.

Conflicts of Interest: The authors declare no conflict of interest. 


\section{References}

1. Ewins, D.J. Control of vibration and resonance in aero engines and rotating machinery-An overview. Int. J. Press. Vessel. Pip. 2010, 87, 504-510. [CrossRef]

2. Behzad, M.; Alvandi, M.; Mba, D.; Jamali, J. A finite element-based algorithm for rubbing induced vibration prediction in rotors. J. Sound Vib. 2013, 332, 5523-5542. [CrossRef]

3. Hong, J.; Zhang, Z.; Xu, X.; Ma, Y.-H. Reliability model of aero-engine structural system. J. Aerosp. Power 2016, 31, 1897-1904.

4. Wang, C.; Hong, Y.; Zhang, W. Object-oriented aero-engine assembly models. Comput. Integr. Manuf. Syst. 2010, 16, 942-948. [CrossRef]

5. Mitra, M.; Bogdan, I.E. Dynamic Modeling and Projection-Based Reduction Methods for Bladed Disks with Nonlinear Frictional and Intermittent Contact Interfaces. Appl. Mech. Rev. 2019, 71, 050803. [CrossRef]

6. Cui, Y.; Deng, S.; Zhang, W. The impact of roller dynamic unbalance of high-speed cylindrical roller bearing on the cage nonlinear dynamic characteristics. Mech. Mach. Theory. 2017, 118, 65-83.

7. Wang, D.; Wang, N.; Chen, K. Unbalance response of a magnetic suspended dual-rotor system. Proc. Inst. Mech. Eng. Part G J. Aerosp. Eng. 2019, 233, 5758-5772. [CrossRef]

8. Sun, C.; Chen, D.; Li, C.; Liu, Y.; Liu, Z.; Hu, M.; Tan, J. A novel constrained optimization-build method for precision assembly of aircraft engine. Assem. Autom. 2019. [CrossRef]

9. Zhang, T.Y.; Zhang, Z.J.; Jin, X. An innovative method of modeling plane geometric form errors for precision assembly. Proc. Inst. Mech. Eng. Part B J. Eng. Manuf. Vol. 2016, 230, 1087-1096. [CrossRef]

10. Li, Y.; Zhang, L.; Wang, Y. An optimal method of posture adjustment in aircraft fuselage joining assembly with engineering constraints. Chin. J. Aeronaut. Vol. 2017, 30, 2016-2023. [CrossRef]

11. Sun, C.; Liu, Z.; Liu, Y.; Wang, X.; Tan, J. An Adjustment Method of Geometry and Mass Centers for Precision Rotors Assembly. IEEE Access 2019, 7, 169992-170002. [CrossRef]

12. Lusty, C.; Keogh, P. Active Vibration Control of a Flexible Rotor by Flexibly Mounted Internal-Stator Magnetic Actuators. IEEE-ASME Trans. Mechatron. 2018, 23, 2870-2880. [CrossRef]

13. Maldonado, D.J.G.; Karev, A.; Hagedorn, P.; Ritto, T.G.; Sampaio, R. Analysis of a rotordynamic system with anisotropy and nonlinearity using the Floquet theory and the method of normal forms. J. Sound Vib. 2019, 453, 201-213. [CrossRef]

14. Glasgow, D.A.; Nelson, H.D. Stability analysis of rotor-bearing systems using component mode synthesis. J. Mech. Des. 1980, 102, 352-359. [CrossRef]

15. Lalanne, M.; Ferraris, G. Rotordynamics "Prediction in Engineering"; Wiley: New York, NY, USA, 1998.

16. Wang, N.; Liu, C.; Jiang, D.; Behdinan, K. Casing vibration response prediction of dual-rotor-blade-casing system with bladecasing rubbing. Mech. Syst. Signal Process. 2019, 118, 61-77. [CrossRef]

17. Chiang, H.W.D.; Hsu, C.N.; Tu, S.H. Rotor-bearing analysis for turbomachinery single-and dual-rotor systems. J. Propuls. Power 2004, 20, 1096-1104. [CrossRef]

18. Wang, N.; Jiang, D.; Xu, H. Effects of Rub-Impact on Vibration Response of a Dual-Rotor System-Theoretical and Experimental Investigation. Exp. Tech. 2019, 44, 299-311. [CrossRef] 